VOLUME 27 (2020) 147-168

DOI: $10.24330 /$ ieja.662998

\title{
ON THE TOPOLOGICITY OF CATEGORIES OF COALGEBRAS
}

\author{
Maurice Kianpi \\ Received: 13 March 2019; Revised: 31 October 2019; Accepted: 5 November 2019 \\ Communicated by Abdullah Harmancı
}

\begin{abstract}
Concrete categories of functorial coalgebras are derived from given concrete categories under a certain commutativity condition satisfied by the underlying forgetful functor and endofunctors of its domain and codomain. When the base category is topological, so is that of functorial coalgebras when in addition to the commutativity condition the endofunctor of its domain preserves initial sources. We investigate the connection between fibres of objects in the topological category of coalgebras and those of the topological base category as well as some generalizations of the coalgebraic topological functor.
\end{abstract}

Mathematics Subject Classification (2010): 18A35, 18A40, 55U40

Keywords: Concrete category, functor, coalgebra, fibre, topological category

\section{Introduction}

Categories of structures with which general topology concerns itself are categories $\mathrm{C}$ together with an underlying functor $T: \mathrm{C} \rightarrow \mathrm{X}$ which is a forgetful functor, where $\mathrm{X}$ is usually the category of sets or some other algebraic category. The common and characteristic feature of all these categories is that $C$ admits initial structures with respect to $T$. The category $\mathrm{C}$ is then concrete over $\mathrm{X}$, since in this case $T$ is faithful (see $[2,4,5,6,7,8,9,10])$ and the pair (C, $T)$ is then called a topological category over $\mathrm{X}$, as well as the functor $T$. When $\mathrm{C}$ is taken to be the category $\operatorname{Alg}(\Sigma)$ of algebras over a given signature $\Sigma$ and $X$ is that of sets and mappings, the forgetful functor to sets does not have this property unless $\Sigma=\emptyset$ [2, Example 21.20]. Therefore, generally the forgetful functor from a category of algebras of an endofunctor of a given category to itself is not topological. This is the reason why in this paper we find conditions under which a category of coalgebras over a topological category is topological too.

Intuitively topological functors are relativised complete preorders. This can be seen in many places throughout the literature: for example, in the various completion processes by which a faithful functor may be turned into a topological one [8], which corresponds to the constructions by which a poset may be turned into a complete lattice, and indeed reduce to these constructions when $X=1[6]$. From a 
topological functor one can obtain many others. For instance, when $T$ is topological and $J$ is a category, then so is the induced functor $T^{J}: C^{J} \rightarrow X^{J}[14]$. Moreover, in [18], it is shown that one of the conditions under which the category $C$ inherits from a category $X$ the property that all descent morphisms in $X$ are effective is that $\mathrm{C}$ be regular, $\mathrm{X}$ have coequalizers and there exists a topological functor $\mathrm{C} \rightarrow \mathrm{X}$. Moreover, topological functors are faithful, amnestic, transportable and have left and right adjoints $[2,5]$. In this paper some of the results that we obtain are based on Strong $\mathfrak{M}$ ono-topological categories (in the coalgebraic setting), where Strong Mono denotes the conglomerate of all strong mono-sources. Monotopological categories were introduced by Nel in [15] as initially structured categories, as a generalization of topological categories in order to include other important categories, such as the category Haus of Hausdorff spaces (and continuous maps). Using results of Herrlich from [7], Schwarz has shown in [17] that a category is monotopological if and only if it is (concretely isomorphic to) an epi-reflective subcategory of some topological category [16]. M-topological functors are well-known examples of so-called topologically algebraic functors which were introduced by Y.H. Hong $[10,11]$ as a generalization of topological functors and which aimed at the study of topological algebras [5]. The results that we obtain here concerning categories of coalgebras, for instance the main one showing that provided that a topological functor and endofunctors of both its domain and its codomain satisfy some reasonable conditions the functor induced between corresponding categories of coalgebras is also topological, yield new tools for future investigations in the framework of topological categories.

The paper is organized as follows. Section 2 is devoted to preliminaries. Here, we give basic concepts and results that will be needed throughout our work and deal with factorization structures in categories of coalgebras. We show that in case $\mathrm{C}$ is an ( $\mathcal{E}$ pi, Strong $\mathfrak{M o n o})$-category and $F: \mathcal{C} \rightarrow \mathrm{C}$ is a functor which preserves strong mono-sources, then so is the category $C_{F}$ of $F$-coalgebras and homomorphisms between them. In Section 3, we investigate topological functors, topological categories and give some basic definitions and results. In particular, we show that every topological functor preserves strong mono-sources, and hence strong monos. A functor $T: \mathrm{C} \rightarrow \mathrm{X}$ and endofunctors $F$ and $G$ of $\mathrm{C}$ and $\mathrm{X}$, respectively, are said to satisfy the global commutativity if $G \circ T=T \circ F$. We investigate endofunctors that preserve intiality as well as properties of concrete categories of coalgebras, most of which are related to the global commutativity, and we find and build examples of such endofunctors. These are the major conditions needed to obtain topological categories of coalgebras. Section 4 is the bare-bone of 
the paper. Here we show that if $T: \mathrm{C} \rightarrow \mathrm{X}$ is a topological functor and $F$ and $G$ are endofunctors of $\mathrm{C}$ and $\mathrm{X}$, respectively, satisfying the global commutativity and such that $F$ preserves $T$-initial sources, then the functor $T_{F, G}: \mathrm{C}_{F} \rightarrow \mathrm{X}_{G}$ that associates $\varphi:(A, \alpha) \rightarrow(B, \beta)$ with $T(\varphi):(T(A), T(\alpha)) \rightarrow(T(B), T(\beta))$ is also topological. We also discuss the transfer of completeness from underlying topological categories to those of coalgebras and investigate the connection between fibres of objects in the topological category of coalgebras and those of the topological base category. To end this section and the paper as well, we find conditions under which the functor $T_{F, G}$ is Strong $\mathfrak{M}$ ono-topological.

\section{Preliminaries}

All categories in this paper are to be considered in the sense of [2]. Here and throughout the paper, $\mathrm{C}$ is a category and $F: \mathrm{C} \rightarrow \mathrm{C}$ a functor. In this section we recall some definitions and a few things that we need to refer to.

A coalgebra (of type $F$ ) is a pair $\mathcal{A}=(A, \alpha: A \rightarrow F(A))$. $\alpha$ (resp. A) is called the cooperation, structure morphism or dynamics (resp. carrier) of $(A, \alpha)$. An F-morphism (or a morphism) from $(A, \alpha)$ to $(B, \beta)$ is a morphism $\varphi: A \rightarrow B$ such that $F(\varphi) \circ \alpha=\beta \circ \varphi$. With morphisms between them, coalgebras form a category [1,3]. Denote it by $\mathrm{C}_{F}$ and by $U_{F}: \mathrm{C}_{F} \rightarrow \mathrm{C}$ the coalgebraic forgetful functor sending $\varphi:(A, \alpha) \rightarrow(B, \beta)$ to $\varphi: A \rightarrow B$. $C_{F}$ has all colimits that exist in $\mathrm{C}$ and all limits that are preserved by $F$, all of which are created by $U_{F}$. Thus in particular epis (isos) in the former are carried by epis (isos) in the latter [1]. When $U_{F}$ has a right adjoint (or, equivalently, $F$ generates a cofree comonad, or, $U_{F}$ is comonadic (see e.g. [12])), $F$ is called a covarietor, see e.g. [1,3].

Given a category $\mathbf{X}$, a concrete category over $\mathbf{X}$ is a pair $(\mathrm{C}, \mathrm{T})$, where $T: \mathrm{C} \rightarrow \mathrm{X}$ is a faithful functor. Sometimes $T$ is called the forgetful (or underlying) functor of the concrete category and $\mathbf{X}$ is called the base category for $(\mathrm{C}, \mathrm{T})$. In case $\mathbf{X}=$ Set, $(C, T)$ is called a construct. In the sequel, the results about concrete categories in the abstract setting and all the definitions can be found, for instance, in $[2,5,9]$. Examples of concrete categories abound in [2, Examples 5.2] and [5]. Because of the faithfulness of $T$, for all morphism $f: A \rightarrow B$ in $\mathrm{C}$, the morphism $T(f): T(A) \rightarrow$ $T(B)$ will also be denoted by $f$. An object $A$ in $\mathrm{C}$ is called discrete whenever, for each object $B$, every morphism $T(A) \rightarrow T(B)$ is a morphism in C. Likewise, $A$ is called indiscrete whenever, for each object $B$, every morphism $T(B) \rightarrow T(A)$ is a morphism in C.

The fibre of an object $X$ in $\mathrm{X}$ is the preordered class consisting of all objects $A$ in $C$ with $T(A)=X$ ordered by: $A \leq B$ if and only if $i d_{X}: T(A) \rightarrow T(B)$ is 
a morphism in C. Objects $A$ and $B$ in $\mathrm{C}$ are said to be equivalent provided that $A \leq B$ and $B \leq A$.

Recalling that a partially ordered class $(L, \leq)$ is called a large complete lattice provided that every subclass of $L$ has a meet and a join, $(C, T)$ is said to be fibrecomplete provided that its fibres are (possibly large) complete lattices. It is called fibre-small provided that each of its fibres is small, i.e., a preordered set, and it is said to be fibre-discrete provided that its fibres are ordered by equality.

A functor $T: \mathrm{C} \rightarrow \mathrm{X}$ is said to reflect identities provided that for every object $A$ and every morphism $k$ in $\mathrm{C}$, if $T(k)$ is an identity in $\mathrm{X}$, then $k$ must be an identity in $\mathrm{C}$ and $T$ (resp. (C, $T$ ) for $T$ faithful) is called amnestic provided that if $k$ is an iso in $\mathrm{C}$ and $T(k)$ is an identity in $\mathrm{X}$, then $k$ must be an identity in $\mathrm{C}$ (resp. the fibres of $T$ are partially ordered classes; i.e., no two different objects in $\mathrm{C}$ are equivalent). Likewise, $(\mathrm{C}, T)$ is said to be (uniquely)transportable provided $T$ is so; i.e., for every object $A$ in $\mathrm{C}$ and every iso $k: T(A) \rightarrow X$ in $\mathrm{X}$, there exists a (unique) object $B$ in C with $T(B)=X$ such that $k: A \rightarrow B$ is an iso in C. For example, the skeleton of the category $V e c$ (of real vectors spaces and homomorphisms between them) formed by all spaces $\mathbb{R}^{m}$ is a construct which is amnestic but not transportable, see [2] from which the following can be extracted:

Proposition 2.1. A concrete category $(C, T)$ over $X$ is fibre-discrete if and only if $T$ reflects identities. Moreover, a concrete category is uniquely transportable if and only if it is transportable and amnestic.

It is clear that a functor that reflects identities is amnestic. Thus for a concrete category, fibre-discrete implies amnestic. The following is immediately checked:

Example 2.2. The concrete category $\left(C_{F}, U_{F}\right)$ over $\mathrm{C}$ is fibre-discrete, hence amnestic. Also, $U_{F}$ reflects both isos and identities and is transportable, hence it is uniquely transportable by Proposition 2.1. Moreover, for any object $A$ in C, there exists a bijective correspondence between the underlying class of the fibre of $A$ and the set $\operatorname{Hom}(A, F(A))$. Hence $\left(C_{F}, U_{F}\right)$ is fibre-small. It is fibre-complete just in case each fibre has one element, i.e., $|\operatorname{Hom}(A, F(A))|=1$ for each object $A$ in $\mathrm{C}$.

Let $T: \mathrm{C} \rightarrow \mathrm{X}$ be a functor and $X \in \mathrm{X}$.

A $T$-structured arrow with domain $X$ is a pair $(f, C)$ consisting of an object $C \in \mathrm{C}$ and a morphism $f: X \rightarrow T(C)$. A $T$-structured source is a pair $\left(X,\left(f_{i}, C_{i}\right)_{i \in I}\right)$ that consists of an object $X \in \mathrm{X}$ and a family of $T$-structured arrows $f_{i}: X \rightarrow T\left(C_{i}\right)$ with domain $X$, indexed by some class $I$. A source $\mathcal{S}=\left(f_{i}: C \rightarrow C_{i}\right)_{i \in I}$ in $\mathrm{C}$ is 
called T-initial provided that for each source $\mathcal{T}=\left(g_{i}: A \rightarrow C_{i}\right)_{i \in I}$ in $\mathrm{C}$ and each morphism $h: T(A) \rightarrow T(C)$ with $T(\mathcal{T})=T(\mathcal{S}) \circ h$ there exists a unique morphism $\bar{h}: A \rightarrow C$ in $\mathrm{C}$ with $\mathcal{T}=\mathcal{S} \circ \bar{h}$ and $h=T(\bar{h})$.
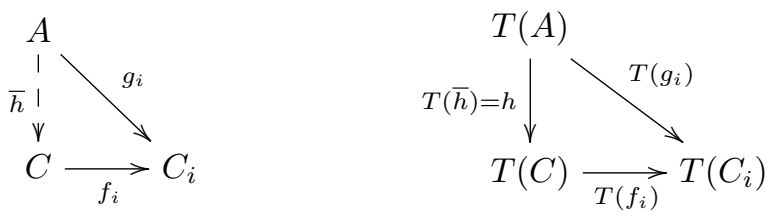

By a $T$-initial morphism in $\mathrm{C}$ is meant a 1 -source $f: A \rightarrow B$ in $\mathrm{C}$ that is $T$-initial. The dual notions are those of $T$-costructured arrow, $T$-costructured sink, $T$-final sink and T-final morphism.

Remark 2.3. ([2, Examples 10.58]) (1) If $(\mathrm{C}, T)$ is a concrete category over $\mathrm{X}$, then $T$-initial sources in $\mathrm{C}$ are precisely initial sources in $(\mathrm{C}, T)$.

(2) Let 1 denote the category consisting of one object and one morphism. Then for the unique functor $T: \mathrm{C} \rightarrow 1, T$-initial sources are precisely the products in $\mathrm{C}$.

Let $T: \mathcal{C} \rightarrow \mathrm{X}$ be a functor, $\mathcal{E}$ a class of $T$-structured arrows and $\mathfrak{M}$ a conglomerate (i.e. a collection) of sources in $\mathrm{C}$ both closed under composition with isomorphisms. The notions of factorization structure $(\mathcal{E}, \mathfrak{M})$ for $T$ and equivalently of $T$ being an $(\mathcal{E}, \mathfrak{M})$-functor are to be considered in the sense of $[2,5,9]$. Thus each $T$-structured source decomposes into an $\mathcal{E}$-morphism followed by a $T$ structured $\mathfrak{M}$-source, and $T$ has the unique $(\mathcal{E}, \mathfrak{M})$-diagonalization property; i.e., whenever $f: X \rightarrow T(A)$ and $e: X \rightarrow T(B)$ are $T$-structured arrows with $e \in \mathcal{E}$ and $\left(m_{i}: A \rightarrow A_{i}\right)_{i \in I} \in \mathfrak{M}$ and $\left(f_{i}: B \rightarrow A_{i}\right)_{i \in I}$ are sources in $\mathrm{C}$, such that $\left(\left(T\left(m_{i}\right) \circ f\right)=\left(T\left(f_{i}\right)\right) \circ e\right.$ for each $i \in I$, then there exists a unique diagonal, i.e., a morphism $d: B \rightarrow A$ with $f=(T(d)) \circ e$ and $f_{i}=m_{i} \circ d$ for each $i \in I$. C is an $(\mathcal{E}, \mathfrak{M})$-category if and only if $I d_{\mathcal{C}}$ is an $(\mathcal{E}, \mathfrak{M})$-functor.

Let (Strong) Mono denote the conglomerate of all (strong) mono-sources in a given category. This means that $\mathfrak{M}$ ono consists of all sources $\mathcal{S}=\left(m_{i}: X \rightarrow X_{i}\right)_{i \in I}$ such that $m_{i} \circ f=m_{i} \circ g$ for all $i \in I$ implies $f=g$, and Strong Mono consists of all mono-sources $\mathcal{S}=\left(m_{i}: X \rightarrow X_{i}\right)_{i \in I}$ such that when we have an epi $e: Y \rightarrow Z$, a morphism $g: Y \rightarrow X$ and a source $\mathcal{R}=\left(h_{i}: Z \rightarrow X_{i}\right)_{i \in I}$ with $\mathcal{S} \circ g=\mathcal{R} \circ e$, then there is a (unique) morphism $t: Z \rightarrow X$ with $t \circ e=g$ and $\mathcal{S} \circ t=\mathcal{R}$. A 1-strong mono-source in $\mathrm{C}$ is called a strong mono. Set is an ( $\mathcal{E}$ pi, Mono)-category, and this is the only nontrivial factorization structure for Set [2, Example 15.3]. Since in Set $\mathfrak{M}$ ono $=\mathfrak{S}$ trong $\mathfrak{M}$ ono, it is an $(\mathcal{E}$ pi, Strong $\mathfrak{M}$ ono)-category. 
Observe that for $\mathcal{E}=\mathcal{E} p i$ and $\mathfrak{M}=\mathfrak{S}$ trong $\mathfrak{M}$ ono, if the category has $(\mathcal{E}, \mathfrak{M})$ factorizations, then it is automatically an $(\mathcal{E}, \mathfrak{M})$-category.

Lemma 2.4. If $\mathcal{C}$ is an (E $\mathcal{E}$ i, $\mathfrak{S}$ trong $\mathfrak{M}$ ono)-category and $F$ preserves strong monosources, then strong mono-sources in $C_{F}$ are precisely the sources carried by strong mono-sources in $C$.

Proof. Let $\mathcal{S}=\left(m_{i}:(A, \alpha) \rightarrow\left(A_{i}, \alpha_{i}\right)\right)_{i \in I}$ be a source in $\mathrm{C}_{F}$. Assume that $U_{F}(\mathcal{S})$ is a strong mono-source in $\mathrm{C}$ and let $h:(C, \gamma) \rightarrow(D, \delta), f:(C, \gamma) \rightarrow(A, \alpha)$, and $\mathcal{K}=\left(d_{i}:(D, \delta) \rightarrow\left(A_{i}, \alpha_{i}\right)\right)_{i \in I}$ be an epi, a morphism and a source in $\mathrm{C}_{F}$, respectively, such that $\mathcal{K} \circ h=\mathcal{S} \circ f$. Then one has $U_{F}(\mathcal{K}) \circ h=U_{F}(\mathcal{S}) \circ f$ with $h$ an epi in C. Thus there is a diagonal fill-in $d: D \rightarrow A$ in $\mathrm{C}$ such that $d \circ h=f$ and $U_{F}(\mathcal{S}) \circ d=U_{F}(\mathcal{K})$. Since $h$ is an epi in C, one easily checks that $d:(D, \delta) \rightarrow(A, \alpha)$ is a morphism in $\mathcal{C}_{F}$ and that $\mathcal{S} \circ d=\mathcal{K}$. Conversely, if $\mathcal{S}$ is a strong mono-source in $\mathrm{C}_{F}$, factorize $U_{F}(\mathcal{S})$ in $\mathrm{C}$ as an epi $e: A \rightarrow B$ followed by a strong mono-source $\mathcal{M}=\left(t_{i}: B \rightarrow A_{i}\right)_{i \in I}$. Since $F$ preserves strong mono-sources, $F(\mathcal{M}):=\left(F\left(t_{i}\right): F(B) \rightarrow F\left(A_{i}\right)\right)_{i \in I}$ is a strong mono-source in C. Moreover by setting $\mathcal{R}:=\left(\alpha_{i} \circ t_{i}: B \rightarrow A_{i}\right)_{i \in I}$, one has $\mathcal{R} \circ e=F(\mathcal{M}) \circ F(e) \circ \alpha$. Thus the diagonal fill-in yields a unique morphism $\beta: B \rightarrow F(B)$ such that $\beta \circ e=F(e) \circ \alpha$ and $F(\mathcal{M}) \circ \beta=\mathcal{R}$; i.e., $\beta \circ e=F(e) \circ \alpha$ and $F\left(t_{i}\right) \circ \beta=\alpha_{i} \circ t_{i}$ for every $i \in I$. In other words, $e:(A, \alpha) \rightarrow(B, \beta)$ is an epi and $\overline{\mathcal{M}}:=\left(t_{i}:(B, \beta) \rightarrow\left(A_{i}, \alpha_{i}\right)\right)_{i \in I}$ is a source in $C_{F}$ carried by a strong mono-source in $C$ such that $\mathcal{S}=\overline{\mathcal{M}} \circ e$. To end the proof, it suffices to show that $e$ is an iso in $C_{F}$. Since $\mathcal{S}$ is a strong mono-source in $\mathrm{C}_{F}$, the last equality which can also be rewritten as $\mathcal{S} \circ i d_{(A, \alpha)}=\overline{\mathcal{M}} \circ e$ yields a unique diagonal fill-in $u:(B, \beta) \rightarrow(A, \alpha)$ in $C_{F}$ such that $u \circ e=i d_{(A, \alpha)}$ and $\mathcal{S} \circ u=\overline{\mathcal{M}}$. Thus $e$ is also a split mono. Hence it is an iso in $\mathrm{C}_{F}$.

Corollary 2.5. If $C$ is an (E pi, Strong $\mathfrak{M}$ ono)-category and $F$ preserves strong

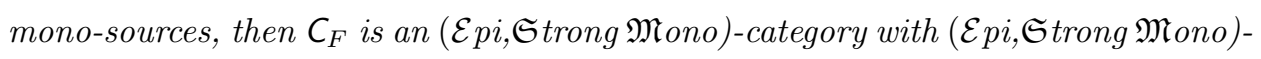
factorizations created by the forgetful functor $U_{F}$.

Proof. Epis in $C_{F}$ are carried by epis in $C$ and by Lemma 2.4 strong mono-sources in $C_{F}$ are carried by strong mono-sources in $C$. Thus since isos in $C_{F}$ are also carried by isos in $\mathrm{C}$ and both $\mathcal{E} p i$ and $\mathfrak{S}$ trong $\mathfrak{M}$ ono are closed under composition with isos in $\mathrm{C}$, so they are in $\mathrm{C}_{F}$, and the category $\boldsymbol{C}_{F}$ has $(\mathcal{E}$ pi, $\mathfrak{S}$ trong $\mathfrak{M o n o})$-factorizations. The unique ( $\mathcal{E}_{\text {pi, }}, \mathfrak{S t r o n g} \mathfrak{M}$ ono)-diagonalization property is immediate from the definition of Strong $\mathfrak{M}$ ono-sources.

Remark 2.6. In case $\mathrm{C}=$ Set, every mono is strong and setting $F^{\prime}(X)=F(X)$ if $X \neq \emptyset$ and $F^{\prime}(\emptyset)=\emptyset$ yields a functor $F^{\prime}$ preserving monos and the categories $\operatorname{Set}_{F}$ 
and $\operatorname{Set}_{F^{\prime}}$ are isomorphic. Moreover strong monos are just 1-strong mono-sources. Thus we retrieve the following well-known results (see [1, Lemmas 4.11 and 4.12 and Corollary 4.13]) in case $\mathrm{C}$ has (epi, strong mono)-factorizations:

(1) If $F$ preserves strong monos, then strong monos in $C_{F}$ are precisely the morphisms carried by strong monos in $\mathrm{C}$. In particular, in case $\mathrm{C}=\mathrm{Set}$, the strong monos in Set $_{F}$ are precisely the one-to-one homomorphisms.

(2) If $C=$ Set or if $F$ preserves strong monos, then $C_{F}$ has (epi,strong mono)factorizations. They are, in fact, created by forgetful functor $U_{F}$.

\section{Topological functors, topological categories and coalgebras}

3.1. Some basic definitions and results. A functor $T: \mathrm{C} \rightarrow \mathrm{X}$ is called topolog$i c a l$ provided that every $T$-structured source $\left(f_{i}: X \rightarrow T\left(C_{i}\right)\right)_{i \in I}$ in $\mathrm{X}$ has a unique $T$-initial lift $\left(\overline{f_{i}}: C \rightarrow C_{i}\right)_{i \in I}$. When the uniqueness condition is dropped, one says that $T$ is a weak topological functor [14]. A concrete category (C,T) is called topological provided that $T$ is topological. In this case, there are fully faithful functors $T_{1}$ (the discrete functor) and $T_{2}$ (the indiscrete functor) with $T_{1} \dashv T \dashv T_{2}$ and $T \circ T_{1}=i d_{\mathrm{X}}=T \circ T_{2}[2$, Proposition 21.12]. Likewise, given a conglomerate $\mathfrak{M}$ of sources in $\mathrm{X}$, a concrete category $(\mathrm{C}, \mathrm{T})$ over an $(\mathcal{E}, \mathfrak{M})$-category $\mathrm{X}$ is called $\mathfrak{M}$ topological provided that $T$ is so; i.e., every $T$-structured source in $\mathfrak{M}$ has a unique $T$-initial lift (see [2, Exercise 21K]). [2, Theorem 21.40] gives a characterization of such categories. In case $\mathfrak{M}=\mathfrak{M o n o}$ it is called monotopological.

Example 3.1. (1) The constructs Top (of topological spaces and continuous mappings), Rel (of relations and relations preserving mappings), Unif (of uniform spaces and uniformly continuous functions), PMet (of pseudometric spaces and contractions) and Prost (of preordered sets and order preserving mappings) are topological. Moreover, the category TopGrp (of topological groups and continuous homomorphisms) is topological if it is considered as concrete over Grp but it is not so over Top or Set. Considered as a concrete category over Top, the category Unif is $\mathfrak{M}$ topological, where $\mathfrak{M}$ is the collection of initial mono-sources. The constructs HUnif (of separated uniform spaces) and Pos (of posets and order preserving mappings) are monotopological [2, Examples 21.8 and 21.39].

(2) If $\mathrm{D}$ is a small category in the sense of [6] with finite limits, and $T: C \rightarrow X$ is a topological functor, then so is $T \circ-: \operatorname{Lex}(D, C) \rightarrow \operatorname{Lex}(D, X)$. So, as is the forgetful functor from TopGrp to Grp, that from TopVec (of topological vector spaces and continuous homomorphisms) to Vec is topological [6, Examples 2.2]. 
(3) Examples of topological categories in the fuzzy setting can be found in Section 3 of [14].

Remark 3.2. Cotoplogical is the categorical dual of topological; i.e., a functor $T: \mathrm{C} \rightarrow \mathrm{X}$ is called cotopological provided that every $T$-costructured sink $\left(f_{i}\right.$ : $\left.T\left(C_{i}\right) \rightarrow X\right)_{i \in I}$ has a unique $T$-final lift $\left(\overline{f_{i}}: C_{i} \rightarrow C\right)_{i \in I}$. By a result of Antoine [4], the functor $T$ is topological if and only if it is cotopological; i.e., $T: \mathbf{C} \rightarrow \mathbf{X}$ is topological if and only if its opposite $T^{o p}: \mathrm{C}^{o p} \rightarrow \mathrm{X}^{o p}$ is so. See e.g. [5, Theorem 4.1], [2, Theorem 21.9], [6, Remark 2.3]. This is known as the topological duality theorem. In the case $\mathbf{X}=1$, it reduces to the result that a preorder admits all joins if and only if it admits all meets [6]. Topological functors are faithful, uniquely transportable and amnestic, see e.g., [5, Theorem 2.2], [2, Theorem 21.3 and Proposition 21.5]. Topological functors reflect epis, but need not reflect isos and hence that need not reflect equalizers (or finite products) or extremal epis (i.e. epis that do not factor through any proper subobject of their codomains), as a result of [2, Theorem 19.14, Examples 13.37 (3) and Exercise 13C]. Also they do reflect monos thanks to their faithfulness. Weak topological functors lift and preserve weak limits and weak colimits, see [14] where a characterization thereof is given.

The behavior of topological functors w.r.t strong monos follows from the next result.

Lemma 3.3. Let $(C, T)$ be topological over $X$. Then $T$ preserves strong monosources. Moreover, a mono-source $\mathcal{S}$ in $\mathcal{C}$ that is $T$-initial is a strong mono-source whenever $T(\mathcal{S})$ is so.

Proof. Let $\mathcal{S}=\left(m_{i}: C \rightarrow D_{i}\right)_{i \in I}$ be a strong mono-source in C. Since $T$ is right adjoint, then by [2, Proposition 18.6] it preserves mono-sources. Thus $T(\mathcal{S}):=$ $\left(T\left(m_{i}\right): T(C) \rightarrow T\left(D_{i}\right)\right)_{i \in I}$ is a mono-source in X. Let $e: X \rightarrow Y, u: X \rightarrow T(C)$ and $\mathcal{R}=\left(v_{i}: Y \rightarrow T\left(D_{i}\right)\right)_{i \in I}$ be an epi, a morphism and a source in $\mathrm{X}$, respectively, such that $\mathcal{R} \circ e=T(\mathcal{S}) \circ u$. We need to find a unique morphism $\delta: Y \rightarrow T(C)$ in $\mathrm{X}$ such that $T(\mathcal{S}) \circ \delta=\mathcal{R}$ and $\delta \circ e=u$. Now the topologicity of $T$ yields unique $T$-initial lifts $\bar{u}: A \rightarrow C$ and $\overline{\mathcal{R}}=\left(\overline{v_{i}}: B \rightarrow D_{i}\right)_{i \in I}$ in $\mathrm{C}$, for the $T$-structured 1-source $u: X \rightarrow T(C)$ and the mono-source $\mathcal{R}$, respectively. Thus the morphism $e: T(A) \rightarrow T(B)$ and the source $\mathcal{S} \circ \bar{u}$ in $\mathcal{C}$ are such that $T(\mathcal{S} \circ \bar{u})=T(\bar{R}) \circ e$. Therefore by the $T$-initiality of $\overline{\mathcal{R}}$, there exists a unique morphism $\bar{e}: A \rightarrow B$ such that $T(\bar{e})=e$ and $\overline{\mathcal{R}} \circ \bar{e}=\mathcal{S} \circ \bar{u}$. On the other hand, as a faithful functor $T$ reflects epis. Therefore $\bar{e}$ is an epi in C. Thus, since $\mathcal{S}$ is a strong mono-source in $\mathrm{C}$, there 
exists a unique diagonal fill-in $d: B \rightarrow C$ such that $d \circ \bar{e}=\bar{u}$ and $\mathcal{S} \circ d=\overline{\mathcal{R}}$. It follows that $T(d): Y \rightarrow T(C)$ is a morphism in $\mathrm{X}$ such that $T(d) \circ e=u$ and $T(\mathcal{S}) \circ T(d)=\mathcal{R}$. The uniqueness of $T(d)$ as morphism satisfying the equations $T(d) \circ e=u$ and $T(\mathcal{S}) \circ T(d)=\mathcal{R}$ follows from the fact that $e$ is an epi. Hence $T(d)$ is a good candidate for $\delta$.

Now assume that $\mathcal{N}=\left(n_{i}: C \rightarrow D_{i}\right)_{i \in I}$ is a mono-source in $C$ that is $T$-initial and $T(\mathcal{N})$ is a strong mono-source in $\mathrm{X}$ and let $p: A \rightarrow C, \mathcal{Q}=\left(q_{i}: B \rightarrow D_{i}\right)_{i \in I}$ and $f: A \rightarrow B$ be an epi, a source and a morphism in C, respectively, such that $\mathcal{Q} \circ f=\mathcal{N} \circ p$. As a left adjoint, $T$ preserves all colimits and hence epis. Thus $T(f): T(A) \rightarrow T(B)$ is an epi in $\mathrm{X}$ such that $T(\mathcal{Q}) \circ T(f)=T(\mathcal{N}) \circ T(p)$. Since $T(\mathcal{N})$ is a strong mono-source, there exists a unique diagonal fill-in $\mu: T(B) \rightarrow T(C)$ such that $\mu \circ T(f)=T(p)$ and $T(\mathcal{N}) \circ \mu=T(\mathcal{Q})$. Therefore the $T$-initiality of $\mathcal{N}$ yields a unique morphism $\bar{\mu}: B \rightarrow C$ in $C$ such that $T(\bar{\mu})=\mu$ and $\mathcal{N} \circ \bar{\mu}=\mathcal{Q}$. Now $T(\bar{\mu} \circ f)=T(\bar{\mu}) \circ T(f)=\mu \circ T(f)=T(p)$. Thus the faithfulness of $T$ yields that $\bar{\mu} \circ f=p$. The uniqueness of $\bar{\mu}$ as morphism satisfying the equations $\bar{\mu} \circ f=p$ and $\mathcal{N} \circ \bar{\mu}=\mathcal{Q}$ follows from the fact that $f$ is an epi.

Corollary 3.4. Every topological functor $T: C \rightarrow X$ preserves strong monos and a mono in $C$ that is $T$-initial is a strong mono whenever its image under $T$ is so.

In the rest of this paper, we shall deal with functors $T: \mathrm{C} \rightarrow \mathrm{X}, F: \mathrm{C} \rightarrow \mathrm{C}$ and $G: \mathrm{X} \rightarrow \mathrm{X}$ satisfying two major conditions:

(I) $F$ preserves $T$-initial sources.

(II) $T$ globally commutes with $F$ and $G$, i.e. $G \circ T=T \circ F$.

For this reason, we need to show that functors satisfying these conditions exist and can be built from existing ones. Firstly, we have the following:

Remark 3.5. (1) The class of endofunctors of $C$ that preserve $T$-initial sources contains $I d_{\mathrm{C}}$ and is stable under composition.

(2) The class of pairs $(H, K)$ where $H: \mathrm{C} \rightarrow \mathrm{C}$ and $K: \mathrm{X} \rightarrow \mathrm{X}$ are functors such that $K \circ T=T \circ H$ contains at least $\left(I d_{\mathrm{C}}, I d_{\mathrm{X}}\right)$. Moreover, it is closed under componentwise composition and, if $(H, K)$ lies therein with $H$ and $K$ isos of categories, then so does the pair $\left(H^{-1}, K^{-1}\right)$.

(3) For all functors $T: \mathrm{C} \rightarrow \mathrm{X}$ and $S: \mathrm{X} \rightarrow \mathrm{C}$, the following diagram obviously commutes:

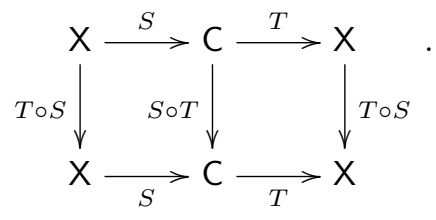


(4) For a functor $F: \mathbf{C} \rightarrow \mathbf{C}$ and a monad $\mathbf{T}: \mathbf{C} \rightarrow \mathrm{C}$, a lifting of $F$ to $\mathrm{C}^{\mathbf{T}}$ is a functor $F^{\mathbf{T}}: \mathrm{C}^{\mathbf{T}} \rightarrow \mathrm{C}^{\mathbf{T}}$ such that $F \circ U^{\mathbf{T}}=U^{\mathbf{T}} \circ F^{\mathbf{T}}$, where $U^{\mathbf{T}}: \mathrm{C}^{\mathbf{T}} \rightarrow \mathrm{C}$ is the forgetful functor.

Let $\mathrm{D}$ be a category with $J$-products for some nonempty set $J$ and $\left(F_{j}: \mathrm{C} \rightarrow\right.$ D) $)_{j \in J}$ a family of functors. For each object $A$ in $\mathrm{C}$, let $p_{j}^{A}: \Pi_{j \in J} F_{j}(A) \rightarrow F_{j}(A)$ denote the $j^{\text {th }}$ canonical projection of the product $\Pi_{j \in J} F_{j}(A)$ of the $F_{j}(A)$ 's in D. Let $f: A \rightarrow B$ be a morphism in C.

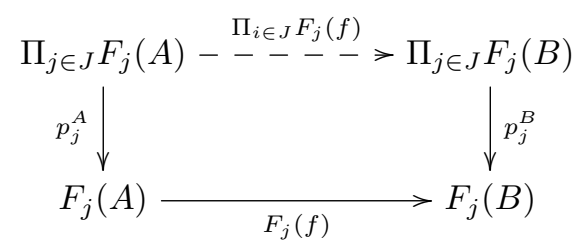

For the source $\left(F_{j}(f) \circ p_{j}^{A}: \Pi_{j \in J} F_{j}(A) \rightarrow F_{j}(B)\right)_{j \in J}$, the universal property of the product yields a unique morphism $\Pi_{i \in J} F_{j}(f): \Pi_{j \in J} F_{j}(A) \rightarrow \Pi_{j \in J} F_{j}(B)$ such that $p_{j}^{B} \circ \Pi_{j \in J} F_{j}(f)=F_{j}(f) \circ p_{j}^{A}$, for each $j \in J$. Obviously the correspondence $\Pi_{i \in J} F_{j}: \mathrm{C} \rightarrow \mathrm{D}$ sending $f: A \rightarrow B$ to $\Pi_{i \in J} F_{j}(f)$ is functorial.

By the Internal Topological Characterization Theorem, a concrete category (C, $T)$ over $\mathrm{X}$ is topological if and only if $T$ lifts limits uniquely and $(\mathrm{C}, T)$ has indiscrete structures; i.e., every object in $\mathrm{X}$ has an indiscrete lift [2, Theorem 21.18]. Thus, the existence of indiscrete structures is a crucial condition for (C, $T$ ) being topological. These include objects of the form $T_{2}(X)$ for any $X$ in $\mathrm{X}$, where $T_{2}$ is right adjoint to $T$. Constant functors with indiscrete value are examples of $T$-initial sources-preserving functors as can be seen through the following that gives a way of generating such functors.

Lemma 3.6. Let $(C, T)$ be concrete over $X$. Then:

(1) Every constant endofunctor of $C$ sends any nonempty source to a T-initial source. If moreover the constant value is indiscrete, then it sends every source to a T-initial source.

(2) If $C$ has $J$-products for some nonempty set $J, T$ preserves $J$-products and $\left(F_{j}: C \rightarrow C\right)_{j \in J}$ is a family of $T$-initial sources-preserving functors, then so is the functor $\prod_{j \in J} F_{j}$.

Proof. (1) Let $B$ be an object in $\mathrm{C}$ and let $C_{B}: \mathrm{C} \rightarrow \mathrm{C}$ be the constant functor with value $B$. Let $\left(f_{i}: A \rightarrow A_{i}\right)_{i \in I}$ be a nonempty source in $\mathrm{C}$ and let $\left(g_{i}: C \rightarrow\right.$ $\left.C_{B}\left(A_{i}\right)\right)_{i \in I}$ be a source in $\mathrm{C}$ and $\rho: T(C) \rightarrow T\left(C_{B}(A)\right)$ a morphism in $\mathrm{X}$ such 
that $T\left(g_{i}\right)=T\left(C_{B}\left(f_{i}\right)\right) \circ \rho$ for each $i \in I$. Then $g_{i}: C \rightarrow B$ for each $i \in I$, $\rho: T(C) \rightarrow T(B)$ and $T\left(g_{i}\right)=T\left(i d_{B}\right) \circ \rho$, i.e., $T\left(g_{i}\right)=\rho$ for each $i \in I$. Then the faithfulness of $T$ yields that for all $i, i^{\prime} \in I, g_{i}=g_{i^{\prime}}$. Let $g$ be the common value of the $g_{i}$ 's. Then $g: C \rightarrow B$ is a morphism in C such that $T(g)=\rho$ and it is unique again because $T$ is faithful. Assume that in addition $B$ is an indiscrete object and $I=\emptyset$. By Remark $2.3 T$-initial sources are precisely initial sources and by [2, Examples 10.42 (1)] empty initial sources are precisely those with an indiscrete base object. Thus in this case $A$ is an indiscrete object and since by assumption its image $B$ under $C_{B}$ is indiscrete too, it follows that $C_{B}$ preserves empty $T$-initial sources.

(2) Let $\left(f_{i}: A \rightarrow A_{i}\right)_{i \in I}$ be a $T$-initial source in C. We want to show that its image $\left(\Pi_{j \in J} F_{j}\left(f_{i}\right): \Pi_{j \in J} F_{j}(A) \rightarrow \Pi_{j \in J} F_{j}\left(A_{i}\right)\right)_{i \in I}$ under the functor $\Pi_{j \in J} F_{j}: \mathrm{C} \rightarrow \mathrm{C}$ is also $T$-initial in C. We first deal with the case $I \neq \emptyset$. Let $\left(g_{i}: C \rightarrow \Pi_{j \in J} F_{j}\left(A_{i}\right)\right)_{i \in I}$

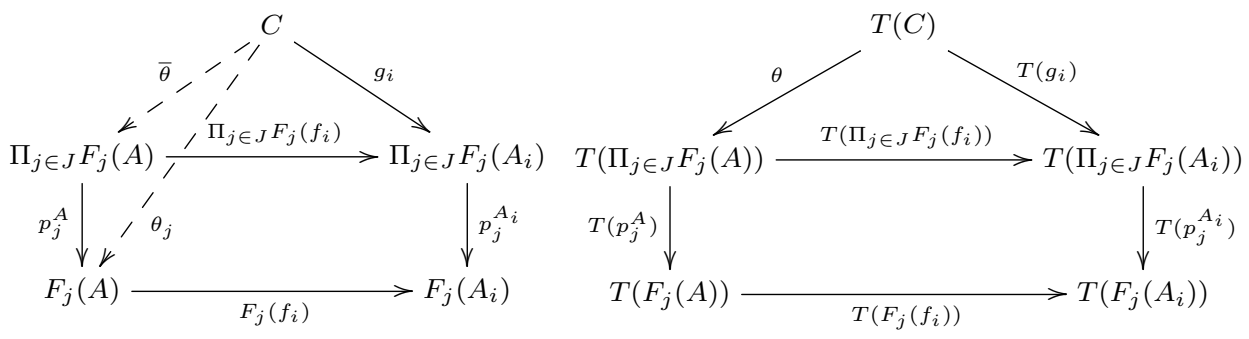

be a source in $\mathrm{C}$ and $\theta: T(C) \rightarrow T\left(\Pi_{j \in J} F_{j}(A)\right)$ a morphism in $\mathrm{X}$ such that $T\left(g_{i}\right)=$ $T\left(\Pi_{j \in J} F_{j}\left(f_{i}\right)\right) \circ \theta$, for each $i \in I$. Fix $j \in J$. Then $T\left(p_{j}^{A}\right) \circ \theta: T(C) \rightarrow T\left(F_{j}(A)\right)$ in $\mathrm{X}$ and for each $i \in I$, by the definition of $\Pi_{j \in J} F_{j}, T\left(F_{j}\left(f_{i}\right)\right) \circ T\left(p_{j}^{A}\right) \circ \theta=$ $T\left(p_{j}^{A_{i}}\right) \circ T\left(\Pi_{j \in J} F_{j}\left(f_{i}\right)\right) \circ \theta=T\left(p_{j}^{A_{i}}\right) \circ T\left(g_{i}\right)$. Thus by the $T$-initiality of the source $\left(F_{j}\left(f_{i}\right): F_{j}(A) \rightarrow F_{j}\left(A_{i}\right)\right)_{i \in I}$, there exists a unique morphism $\theta_{j}: C \rightarrow F_{j}(A)$ in $\mathrm{C}$ such that $F_{j}\left(f_{i}\right) \circ \theta_{j}=p_{j}^{A_{i}} \circ g_{i}$ and $T\left(\theta_{j}\right)=T\left(p_{j}^{A}\right) \circ \theta$. Thus, for the source $\left(\theta_{j}: C \rightarrow F_{j}(A)\right)_{j \in J}$ the universal property of the product in $\mathrm{C}$ yields a unique morphism $\bar{\theta}: C \rightarrow \prod_{j \in J} F_{j}(A)$ such that for each $j \in J$ one has $\theta_{j}=p_{j}^{A} \circ \bar{\theta}$. Fix $i \in I$. Then for each $j \in J$ one has:

$$
\begin{aligned}
& p_{j}^{A_{i}} \circ \prod_{j \in J} F_{j}\left(f_{i}\right) \circ \bar{\theta}=F_{j}\left(f_{i}\right) \circ p_{j}^{A} \circ \bar{\theta} \\
& =F_{j}\left(f_{i}\right) \circ \theta_{j} \\
& \text { and } \\
& T\left(p_{j}^{A}\right) \circ T(\bar{\theta})=T\left(p_{j}^{A} \circ \bar{\theta}\right) \\
& =p_{j}^{A_{i}} \circ g_{i} \\
& =T\left(\theta_{j}\right) \\
& =T\left(p_{j}^{A}\right) \circ \theta .
\end{aligned}
$$

Since the $p_{j}^{A_{i}}$ 's and the $T\left(p_{j}^{A}\right)$ 's are jointly monic in C and $\mathrm{X}$, respectively, it holds that $\Pi_{j \in J} F_{j}\left(f_{i}\right) \circ \bar{\theta}=g_{i}$ and $T(\bar{\theta})=\theta$, respectively.

Now we assume that $I=\emptyset$; i.e., $A$ is an indiscrete object in $\mathrm{C}$ and we show that $\left(\Pi_{j \in J} F_{j}\right)(A):=\Pi_{j \in J} F_{j}(A)$ is so. Let $B$ be an object in $C$ and let $\gamma: T(B) \rightarrow$ 
$T\left(\Pi_{j \in J} F_{j}(A)\right)$ be a morphism. We need to find a morphism $\bar{\gamma}: B \rightarrow \Pi_{j \in J} F_{j}(A)$ in $C$ such that $T(\bar{\gamma})=\gamma$. The proof is essentially the same as above: it suffices to replace $C$ with $B, \theta$ with $\gamma$ and ignore the part concerning the family $\left(g_{i}\right)_{i \in I}$.

For each object $A$ and each nonempty set $J, C_{A} \times(-)_{C}^{J}$ maps every morphism $f: B \rightarrow C$ of $C$ to $i d_{A} \times f^{J}: A \times B^{J} \rightarrow A \times C^{J}$. Moreover, obviously identity functors are $T$-initial sources-preserving. Thus from Lemma 3.6, the following that yields examples of initial sources preserving functors is straightforward:

Corollary 3.7. Let $(C, T)$ be concrete over $X$. For every nonempty set $J$ such that $C$ has $J$-powers, if $T$ preserves $J$-powers, then the functor $(-)_{C}^{J}:=\Pi_{j \in J} F_{j}$ with $F_{j}=I d_{C}$ for each $j \in J$ preserves $T$-initial sources. Moreover, for all object $A$ in $C$, the functor $C_{A} \times(-)_{C}^{J}$ preserves nonempty $T$-initial sources and in case $A$ is indiscrete, that preserves empty ones too.

Example 3.8. Take $C=$ TopGrp and let $T$ be the usual forgetful functor to Grp. Since Grp is (co)complete with products being "direct products" considered as sources via projections, [2, Theorem 21.16] implies that so is TopGrp, and products therein are carried by products in Grp (which in turn are carried by products in Set) since as a right adjoint $T$ preserves all limits. A similar observation holds for TopVec and Vec. Recalling that products in Set may be formed by taking the Cartesian product, let the notation of the form $\Pi_{j \in J} X_{j}$ refer here to the Cartesian product of sets, or to the Cartesian product equipped with the convenient structure. If $\left(F_{j}: \mathrm{C} \rightarrow \mathrm{C}\right)_{j \in J}$ and $\left(G_{j}: \mathrm{X} \rightarrow \mathrm{X}\right)_{j \in J}$ for some nonempty set $J$ are families of functors such that $G_{j} \circ T=T \circ F_{j}$ for each $j \in J$, then $\left(\Pi_{j \in J} G_{j}\right) \circ T=T \circ\left(\Pi_{j \in J} F_{j}\right)$ where $T$ denotes the usual forgetful functor in each of the following cases:

(1) $C$ is a topological construct and $X=$ Set.

(2) $\mathrm{C}=$ TopGrp (resp. Top Vec) and $\mathbf{X}=$ Grp (resp. Vec).

3.2. The functor induced by global commutativity. The correspondence $\mathfrak{F}: \mathrm{C}_{F} \rightarrow \mathrm{C}_{F}$ defined by $(\varphi:(A, \alpha) \rightarrow(B, \beta)) \mapsto(F(\varphi):(F(A), F(\alpha)) \rightarrow$ $(F(B), F(\beta)))$ is functorial and we call it the iteration functor for $F$. Objects in $\left(\mathrm{C}_{F}\right)_{\mathfrak{F}}$ are pairs $((A, \alpha), \theta)$ where $\alpha, \theta: A \rightarrow F(A)$ are morphisms in $\mathrm{C}$ such that $F(\alpha) \circ \theta=F(\theta) \circ \alpha$ and a morphism $\varphi:((A, \alpha), \theta) \rightarrow((B, \beta), \mu)$ in $\left(C_{F}\right)_{\mathfrak{F}}$ is a morphism $\varphi:(A, \alpha) \rightarrow(B, \beta)$ in $\mathrm{C}_{F}$ such that $\mathfrak{F}(\varphi) \circ \theta=\mu \circ \varphi$; i.e., a morphism $\varphi: A \rightarrow B$ in C such that $F(\varphi) \circ \alpha=\beta \circ \varphi$ and $F(\varphi) \circ \theta=\mu \circ \varphi ; \varphi$ is both a morphism from $(A, \alpha)$ to $(B, \beta)$ and from $(A, \theta)$ to $(B, \mu)$. Therefore, there are two coalgebraic forgetful functors from $\left(C_{F}\right)_{\mathfrak{F}}$ to $C_{F}$ namely, $U_{\mathfrak{F}}:(\varphi:((A, \alpha), \theta) \rightarrow$ $((B, \beta), \mu)) \mapsto(\varphi:(A, \alpha) \rightarrow(B, \beta))$ that is the usual coalgebraic forgetful functor 
and the functor $U_{\mathfrak{F}}^{*}:(\varphi:((A, \alpha), \theta) \rightarrow((B, \beta), \mu)) \mapsto(\varphi:(A, \theta) \rightarrow(B, \mu))$. The following is straightforward and stands as the leading ingredient in the sequel:

Lemma 3.9. Let $T: C \rightarrow X$ and $G: X \rightarrow X$ be functors such that $G \circ T=T \circ F$. Then the correspondence $T_{F, G}: C_{F} \rightarrow X_{G}$ defined by $(\varphi:(A, \alpha) \rightarrow(B, \beta)) \mapsto$ $(T(\varphi):(T(A), T(\alpha)) \rightarrow(T(B), T(\beta)))$ is functorial. Moreover, $T_{F, G}$ is faithful (resp. an embedding) whenever $T$ is so, and that is full whenever $T$ is fully faithful.

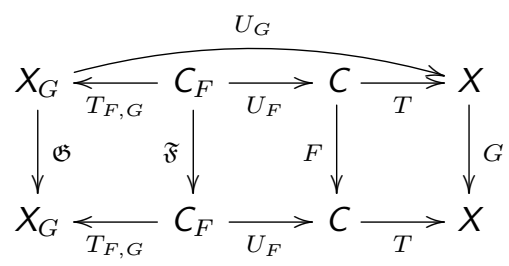

In particular, $\mathfrak{F}=F_{F, F}$. Furthermore, each square in the above diagram is commutative, $U_{\mathfrak{F}}^{*}=\left(U_{F}\right)_{\mathfrak{F}, F}$ and $\left(T \circ U_{F}\right)_{\mathfrak{F}, G}=T_{F, G} \circ\left(U_{F}\right)_{\mathfrak{F}, F}$.

The following yields some other nice properties that the functor $T_{F, G}$ inherits from the functor $T$ :

Theorem 3.10. Let $T: C \rightarrow X$ and let $G: X \rightarrow X$ be functors such that $G \circ T=$ $T \circ F$. If $T$ reflects identities (resp. is amnestic, is transportable or is conservative), then so does (resp. is) the functor $T_{F, G}$. If $T$ is faithful, then the concrete category $\left(C_{F}, T_{F, G}\right)$ over $X_{G}$ is fibre-discrete (resp. amnestic or (uniquely)transportable) whenever the concrete category $(C, T)$ over $X$ is so. Also, $U_{F}$ reflects (in)discrete objects in $(C, T)$.

Proof. (1) In case $T$ reflects identities or isos, then so does $T_{F, G}$ since by Lemma 3.9, $U_{G} \circ T_{F, G}=T \circ U_{F}$ and $U_{F}$ does so by Example 2.2. Assume now that $T$ is amnestic and let $\varphi:(A, \alpha) \rightarrow(B, \beta)$ be an iso in $C_{F}$ such that $T_{F, G}(\varphi)$ is an identity in $\mathrm{X}_{G}$. Then again by taking into account the fact that $U_{G} \circ T_{F, G}=T \circ U_{F}$, one gets that $T(\varphi)$ is an identity in X. Now $\varphi$ iso in $\mathrm{C}_{F}$ implies $\varphi$ is an iso in C. Thus the amnesticity of $T$ implies that $\varphi$ must be an identity in $\mathrm{C}$, hence in $\mathrm{C}_{F}$ too.

Assume that $T$ is transportable and let $(A, \alpha)$ be an object in $\mathrm{C}_{F}$ and let $\varphi$ : $T_{F, G}((A, \alpha)) \rightarrow(X, \xi)$ be an iso in $\mathrm{X}_{G}$. Since $U_{G} \circ T_{F, G}=T \circ U_{F}$ and isos in $\mathrm{X}_{G}$ are carried by isos in $\mathrm{X}, \varphi: T(A) \rightarrow X$ is an iso in $\mathrm{X}$. Therefore the transportability of (C,T) yields a $B$ in $\mathrm{C}$ with $T(B)=X$ and an iso $\bar{\varphi}: A \rightarrow B$ in $\mathrm{C}$ such that $T(\bar{\varphi})=\varphi$. Let $\psi: B \rightarrow A$ be the inverse of $\bar{\varphi}$ and set $\beta:=F(\bar{\varphi}) \circ \alpha \circ \psi$. Then obviously $\bar{\varphi}:(A, \alpha) \rightarrow(B, \beta)$ is an iso in $C_{F}$. To end the proof, we need to show 
that $T(\beta)=\xi$. Now

$$
\begin{aligned}
\xi \circ \varphi & =G(\varphi) \circ T(\alpha) \\
& =G(T(\bar{\varphi})) \circ T(\alpha) \\
& =T(F(\bar{\varphi})) \circ T(\alpha) \\
& =T(F(\bar{\varphi}) \circ \alpha) \\
& =T(\beta \circ \bar{\varphi}) \\
& =T(\beta) \circ T(\bar{\varphi}) \\
& =T(\beta) \circ \varphi
\end{aligned}
$$

and $\varphi$ is an iso and hence right-cancellable. Thus $\xi=T(\beta)$.

(2) Assume that $T$ is faithful and the concrete category $(C, T)$ over $\mathrm{X}$ is fibrediscrete (resp. amnestic or transportable). Then $T$ reflects identities (resp. is amnestic or is transportable) thanks to Proposition 2.1 and [2, Remark 5.6]. Therefore, from the above, $\left(C_{F}, T_{F, G}\right)$ is fibre-discrete (resp. amnestic or transportable) too. Now in this case uniquely transportable is equivalent to amnestic and transportable again by Proposition 2.1. Thus the result about unique transportability also holds. For the last assertion, we give the proof for discrete objects. The indiscrete case is proved analogously. Let $(A, \alpha)$ be an object in $\left(C_{F}, T_{F, G}\right)$ such that $A$ is discrete in $(\mathrm{C}, T)$ and let $(B, \beta)$ be an object in $\mathrm{C}_{F}$ and $\varphi$ : $T_{F, G}((A, \alpha)) \rightarrow T_{F, G}((B, \beta))$ a morphism in $\mathrm{X}_{G}$, i.e., $T(\beta) \circ \varphi=G(\varphi) \circ T(\alpha)$. Since $U_{G} \circ T_{F, G}=T \circ U_{F}$, then by applying $U_{G}$ to $\varphi$, we get that $\varphi: T(A) \rightarrow T(B)$ is a morphism in $\mathrm{X}$ and since $A$ is discrete in $(\mathrm{C}, T)$, there exists a morphism $\bar{\varphi}: A \rightarrow B$ in $\mathrm{C}$ such that $T(\bar{\varphi})=\varphi \cdot \bar{\varphi}$ is in fact a morphism in $\mathrm{C}_{F}$ from $(A, \alpha)$ to $(B, \beta)$. Indeed, $T(\beta) \circ \varphi=G(\varphi) \circ T(\alpha)$ implies $T(\beta) \circ T(\bar{\varphi})=G(T(\bar{\varphi})) \circ T(\alpha)$; i.e. $T(\beta \circ \bar{\varphi})=T(F(\bar{\varphi}) \circ \alpha)$ and the faithfulness of $T$ yields $\beta \circ \bar{\varphi}=F(\bar{\varphi}) \circ \alpha$.

Proposition 3.11. Let $T: C \rightarrow X$ and $G: X \rightarrow X$ be functors with $T$ faithful such that $G \circ T=T \circ F$. If $\left(\varphi_{i}:(A, \alpha) \rightarrow\left(A_{i}, \alpha_{i}\right)\right)_{i \in I}$ is a $T_{F, G}$-initial source in $C_{F}$ and $\left(F\left(\varphi_{i}\right): F(A) \rightarrow F\left(A_{i}\right)\right)_{i \in I}$ is a $T$-initial source in $C$, then the source $\left(\mathfrak{F}\left(\varphi_{i}\right): \mathfrak{F}((A, \alpha)) \rightarrow \mathfrak{F}\left(\left(A_{i}, \alpha_{i}\right)\right)\right)_{i \in I}$ is $T_{F, G}$-initial too. In particular, in case $F$ is a constant functor with an indiscrete value, $\mathfrak{F}$ preserves $T_{F, G}$-initial sources.

Proof. Assume that $\left(\varphi_{i}:(A, \alpha) \rightarrow\left(A_{i}, \alpha_{i}\right)\right)_{i \in I}$ is a $T_{F, G}$-initial source in $\mathrm{C}_{F}$ such that the source $\left(F\left(\varphi_{i}\right): F(A) \rightarrow F\left(A_{i}\right)\right)_{i \in I}$ is $T$-initial in $\mathrm{C}$ and let $\left(\psi_{i}\right.$ : $\left.(C, \gamma) \rightarrow \mathfrak{F}\left(\left(A_{i}, \alpha_{i}\right)\right)\right)_{i \in I}$ be a source in $C_{F}$ and $\theta: T_{F, G}((C, \gamma)) \rightarrow T_{F, G}(\mathfrak{F}((A, \alpha)))$ a morphism in $\mathrm{X}_{G}$ such that $T_{F, G}\left(\mathfrak{F}\left(\varphi_{i}\right)\right) \circ \theta=T_{F, G}\left(\psi_{i}\right)$ for each $i \in I$. Then $T(F(\alpha)) \circ \theta=G(\theta) \circ T(\gamma),\left(\psi_{i}: C \rightarrow F\left(A_{i}\right)\right)_{i \in I}$ is a source in $\mathrm{C}$ and $\theta: T(C) \rightarrow$ 
$T(F(A))$ is a morphism in $\mathbf{X}$ such that $T\left(F\left(\varphi_{i}\right)\right) \circ \theta=T\left(\psi_{i}\right)$ for each $i \in I$. Thus by assumption, there exists a unique morphism $\bar{\theta}: C \rightarrow F(A)$ such that $T(\bar{\theta})=\theta$ and $F\left(\varphi_{i}\right) \circ \bar{\theta}=\psi_{i}$ for each $i \in I$. One has:

$$
\begin{aligned}
T(F(\bar{\theta}) \circ \gamma) & =T(F(\bar{\theta})) \circ T(\gamma) \\
& =G(T(\bar{\theta})) \circ T(\gamma) \\
& =G(\theta) \circ T(\gamma) \\
& =T(F(\alpha)) \circ \theta \\
& =T(F(\alpha)) \circ T(\bar{\theta}) \\
& =T(F(\alpha) \circ \bar{\theta}) .
\end{aligned}
$$

Since $T$ is faithful, it follows that $F(\bar{\theta}) \circ \gamma=F(\alpha) \circ \bar{\theta}$; i.e. $\bar{\theta}:(C, \gamma) \rightarrow \mathfrak{F}((A, \alpha))$ is a morphism in $C_{F}$ such that $T_{F, G}(\bar{\theta})=\theta$ with $F\left(\varphi_{i}\right) \circ \bar{\theta}=\psi_{i}$ in $C_{F}$ for each $i \in I$ and it is unique as such since $T_{F, G}$ is faithful by Lemma 3.9. The last statement follows from Lemma 3.6 (1).

Corollary 3.12. Let $T: C \rightarrow X$ and $G: X \rightarrow X$ be functors with $T$ faithful such that $G \circ T=T \circ F$. If $F$ preserves $T$-initial sources, then $\mathfrak{F}$ preserves $T_{F, G}$-initial sources $\left(\varphi_{i}:(A, \alpha) \rightarrow\left(A_{i}, \alpha_{i}\right)\right)_{i \in I}$ in $C_{F}$ for which $\left(\varphi_{i}: A \rightarrow A_{i}\right)_{i \in I}$ is T-initial in C.

\section{Topologicity of categories of coalgebras}

By Remark 3.2 every topological functor is faithful, amnestic and uniquely transportable but need not be conservative (i.e., need not reflect isos). By Theorem 3.10 the functor $T_{F, G}$ has each of these properties whenever $T$ has, but need not be topological. To be so, in addition every $T_{F, G}$-structured source must have a $T_{F, G^{-}}$ initial lift, by [2, Proposition 21.5]. In this section, it is shown that this holds under some reasonable circumstances so that $T_{F, G}$ inherits topologicity and some of its generalizations from $T$.

4.1. Topological categories of coalgebras. In Section 3 we have given, and shown how to build from existing ones, examples of functors that preserve initial sources as well example of functors that globally commute with pairs of functors. These are the main ingredients that stand as hypothesis in our main theorem.

We start by exhibiting a simple introductory example that serves as motivation. By Remark 2.3 a source in an arbitrary $\mathrm{C}$ is a product if and only if it is $T$-initial, where $T: \mathrm{C} \rightarrow 1$ is the unique functor from $\mathrm{C}$ to 1 . Moreover, $T$ is faithful if $\mathrm{C}$ is a preordered class and a source $\left(p \rightarrow a_{i}\right)_{i \in I}$ is a product (i.e. an initial source) 
if and only if $p=\wedge_{i \in I} a_{i}$. On the other hand, in case $\mathrm{C}$ is a preordered class, an endofunctor thereof is just a correspondence $F: \mathrm{C} \rightarrow \mathrm{C}$ which is order-preserving and an object $a$ carries a coalgebra structure $\alpha: a \rightarrow F(a)$ just in case $a \leq F(a)$. Clearly, $C_{F}$ is also a preordered class and in case $C$ has meets, which amounts to saying that $T$ is topological, so does $C_{F}$ in case $F$ preserves $T$-initial sources; i.e., $F$ preserves meets. This is just an instance of the following:

Theorem 4.1. For every topological functor $T: C \rightarrow X$ and every functor $G: X \rightarrow$ $X$ such that $G \circ T=T \circ F$ and $F$ preserves $T$-initial sources, the functor $T_{F, G}$ is topological. That is, the concrete category $\left(C_{F}, T_{F, G}\right)$ is topological over $X_{G}$.

Proof. Let $\left(\varphi_{i}:(X, \xi) \rightarrow T_{F, G}\left(A_{i}, \alpha_{i}\right)\right)_{i \in I}$ be a $T_{F, G}$-structured source in $\mathrm{X}_{G}$. Then $\left(\varphi_{i}: X \rightarrow T\left(A_{i}\right)\right)_{i \in I}$ is a $T$-structured source in $\mathrm{X}$. Thus the topologicity of $T$ yields a unique $T$-initial lift $\left(\overline{\varphi_{i}}: C \rightarrow A_{i}\right)_{i \in I}$ thereof in $\mathrm{C}$. We have $T\left(\overline{\varphi_{i}}\right)=\varphi_{i}$ and $\left(\alpha_{i} \circ \overline{\varphi_{i}}: C \rightarrow F\left(A_{i}\right)\right)_{i \in I}$ is a source in $C$. On the other hand, $F$ preserves $T$-initial sources. Thus $\left(F\left(\overline{\varphi_{i}}\right): F(C) \rightarrow F\left(A_{i}\right)\right)_{i \in I}$ is a $T$-initial source in C. Moreover, since $\varphi_{i}:(X, \xi) \rightarrow\left(T\left(A_{i}\right), T\left(\alpha_{i}\right)\right)$ is a morphism in $\mathrm{X}_{G}$ for each $i \in I$, it follows that $\xi: X \rightarrow G(X)$ and $T\left(\alpha_{i}\right) \circ \varphi_{i}=G\left(\varphi_{i}\right) \circ \xi($ see $(\mathrm{I})$ ), i.e., $\xi: T(C) \rightarrow T(F(C)$ ) and $T\left(\alpha_{i}\right) \circ T\left(\overline{\varphi_{i}}\right)=G\left(T\left(\overline{\varphi_{i}}\right)\right) \circ \xi$, for each $i \in I$. Thus the $T$-initiality of the source $\left(F\left(\overline{\varphi_{i}}\right): F(C) \rightarrow F\left(A_{i}\right)\right)_{i \in I}$ in $C$ yields a unique morphism $\bar{\xi}: C \rightarrow F(C)$ such that $T(\bar{\xi})=\xi$ and $\alpha_{i} \circ \overline{\varphi_{i}}=F\left(\overline{\varphi_{i}}\right) \circ \bar{\xi}$, for each $i \in I$ (see (II)). We need to show that $\left(\overline{\varphi_{i}}:(C, \bar{\xi}) \rightarrow\left(A_{i}, \alpha_{i}\right)\right)_{i \in I}$ is $T_{F, G}$-initial in $C_{F}$. Let $\left(\psi_{i}:(A, \alpha) \rightarrow\left(A_{i}, \alpha_{i}\right)\right)_{i \in I}$

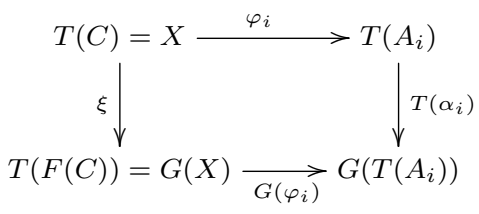

$(I)$

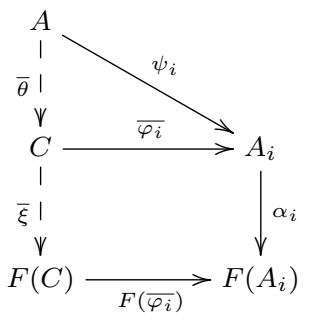

$(I I)$

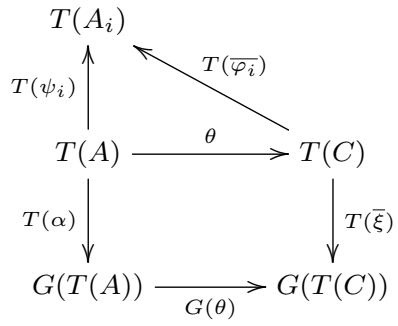

$(I I I)$

be a source in $\mathrm{C}_{F}$ and $\theta: T_{F, G}((A, \alpha)) \rightarrow T_{F, G}(C, \bar{\xi})$ a morphism in $\mathrm{X}_{G}$ such that $T_{F, G}\left(\psi_{i}\right)=T_{F, G}\left(\overline{\varphi_{i}}\right) \circ \theta$ for each $i \in I$. Then $\theta:(T(A), T(\alpha)) \rightarrow(T(C), T(\bar{\xi}))$ and $T\left(\psi_{i}\right)=T\left(\overline{\varphi_{i}}\right) \circ \theta$ for each $i \in I$; i.e., $\theta: T(A) \rightarrow T(C), T(\bar{\xi}) \circ \theta=G(\theta) \circ T(\alpha)$ and $T\left(\psi_{i}\right)=T\left(\overline{\varphi_{i}}\right) \circ \theta$ for each $i \in I$ (see (III)). Therefore the $T$-initiality of the source $\left(\overline{\varphi_{i}}: C \rightarrow A_{i}\right)_{i \in I}$ in C yields a unique $\bar{\theta}: A \rightarrow C$ in $\mathrm{C}$ such that $T(\bar{\theta})=\theta$ and $\overline{\varphi_{i}} \circ \bar{\theta}=\psi_{i}$ for each $i \in I$ (see (II)). Then one has: 


$$
\begin{aligned}
T(F(\bar{\theta}) \circ \alpha) & =T(F(\bar{\theta})) \circ T(\alpha) \\
& =G(T(\bar{\theta})) \circ T(\alpha) \\
& =G(\theta) \circ T(\alpha) \\
& =T(\bar{\xi}) \circ \theta \\
& =T(\bar{\xi}) \circ T(\bar{\theta}) \\
& =T(\bar{\xi} \circ \bar{\theta}) .
\end{aligned}
$$

Now by Remark 3.2 as a topological functor $T$ is faithful. Thus it follows that $F(\bar{\theta}) \circ \alpha=\bar{\xi} \circ \bar{\theta}$. Hence the morphism $\bar{\theta}:(A, \alpha) \rightarrow(C, \bar{\xi})$ is in $\mathrm{C}_{F}$ with $T(\bar{\theta})=\theta$ and $\overline{\varphi_{i}} \circ \bar{\theta}=\psi_{i}$ for each $i \in I$. Its uniqueness as such follows from the faithfulness of $T$.

In case $U_{F}$ is topological, the concrete category $\left(\mathrm{C}_{F}, U_{F}\right)$ is fibre-complete by $[2$, Proposition 21.11], i.e., $|\operatorname{Hom}(A, F(A))|=1$ for each $A$ in $\mathrm{C}$ by Example 2.2. As it has been seen earlier, typical examples of categories in which this happens are thin categories, i.e., preordered classes and these are precisely categories $\mathrm{C}$ for which the unique functor $\mathrm{C} \rightarrow 1$ is faithful. By Lemma 3.9 the equations $U_{G} \circ T_{F, G}=T \circ U_{F}$ and $\left(T \circ U_{F}\right)_{\mathfrak{F}, G}=T_{F, G} \circ\left(U_{F}\right)_{\mathfrak{F}, F}$ hold. Moreover it is well known (see e.g. $[2$, Proposition 21.6]) that topological functors are closed under composition. Hence:

Remark 4.2. (1) If there exists an object $A$ in $\mathrm{C}$ such that $\mid(\operatorname{Hom}(A, F(A)) \mid \geq 2$, then the coalgebraic forgetful functor $U_{F}$ is not topological.

(2) Assume that $F, G$ and $T$ are as in Theorem 4.1. If $U_{F}$ or $U_{G}$ is topological, then so is $T \circ U_{F}$. Likewise, $\left(T \circ U_{F}\right)_{\mathfrak{F}, G}$ is topological whenever $\left(U_{F}\right)_{\mathfrak{F}, F}$ is so.

From Theorem 4.1, Lemma 3.9 and Proposition 3.11 it follows:

Corollary 4.3. Let $T: C \rightarrow X$ be a topological functor. Then the concrete category $\left(C_{I d_{C}}, T_{I d_{C}, I d_{X}}\right)$ is topological over $X_{I d_{X}}$. Assume that $G: X \rightarrow X$ is a functor such that $G \circ T=T \circ F$. If $\mathfrak{F}$ preserves $T_{F, G}$-initial sources, then $\left(T_{F, G}\right)_{\mathfrak{F}, \mathfrak{G}}$ is topological too. In particular this is the case if $F=I d$ or $F=C_{A}$ for some indiscrete object A.

Corollary 3.7, Example 3.8 and Theorem 4.1 yield:

Example 4.4. Let $T: \mathrm{C} \rightarrow \mathrm{X}$ be the usual forgetful functor for $\mathrm{C}$ and $\mathrm{X}$ as in Example 3.8, let $I$ be a nonempty set and $A$ an indiscrete object in $\mathrm{C}$. Then the categories $\left(\mathrm{C}_{C_{A}}, T_{C_{A}, C_{T(A)}}\right)$ and $\left(\mathrm{C}_{A \times(-)_{C}^{I}}, T_{A \times(-)_{C}^{I}, T(A) \times(-)_{\times}^{I}}\right)$ are topological over 
$\mathrm{X}_{C_{T(A)}}$ and $\mathrm{X}_{T(A) \times(-)_{\times}^{I}}$, respectively, and the latter reduces (up to isomorphism) to the topologicity of $\left(\mathrm{C}_{(-)_{C}^{I}}, T_{(-)_{C}^{I},(-)_{C}^{I}}\right)$ over $\mathrm{X}_{(-)_{x}^{I}}$ in case $A$ is a final object.

One of the reasons why topological functors are interesting is that their domains have some properties if and only if their codomains have. These include being (co)complete [2, Theorem 21.16]. As it has been mentioned in Section 2, categories of coalgebras are cocomplete whenever the underlying category is, and that they have all limits that exist and are preserved by the endofunctor. Now it is well known that right adjoints preserve all existing limits. Therefore if $(C, T)$ is topological over $\mathrm{X}$, then:

- for any endofunctor $F$ and $G$ of $\mathrm{C}$ and $\mathrm{X}$, respectively, the categories $\mathrm{C}_{F}$ and $\mathrm{X}_{G}$ are cocomplete if $\mathrm{C}$ or $\mathrm{X}$ is so.

- if $\mathrm{X}$ or $\mathrm{C}$ is complete, then the category $\mathrm{C}_{T_{2} \circ T}$ is complete.

As it is well known, one of the properties that categories of coalgebras hardly have is that of being complete, and hence that of being strongly complete, i.e., both being complete and having intersections. Dual is strongly cocomplete. E.g. Set, Top, Vec, Grp, Hcomp, Pos, where Hcomp is the category of Hausdorff compact topological spaces and continuous mappings are strongly (co) complete [2]. Fortunately, for a strong mono preserving covarietor on a strongly complete category and in particular for a covarietor over Set, the category of coalgebras is complete [1, Theorem 4.14 and Corollary 4.15]. Therefore [2, Theorem 21.16] implies:

Proposition 4.5. Under the assumptions of Theorem 4.1, the category $C_{F}$ is complete if in addition one of the following conditions is satisfied:

(1) $X$ is complete and $G$ preserves limits.

(2) $X$ is strongly complete and $G$ is a covarietor preserving strong monos. In particular if $X=$ Set and $G$ is a covarietor.

Conversely, $X_{G}$ is complete if in (1) or (2) the category $X$ is replaced with $C$ and the functor $G$ with $F$.

Let $T_{F, G}^{-1}(X, \xi)$ (resp. $\left.T^{-1}(X)\right)$ denote the fibre of an object $(X, \xi)$ (resp. $X$ ) in $\mathrm{X}_{G}$ (resp. X). As mentioned earlier, as a topological functor, $T_{F, G}$ (resp. $T$ ) has both left and right adjoints, and these adjoints are fully faithful and are right inverses thereof. Thus $T_{F, G}^{-1}(X, \xi) \neq \emptyset$ (resp. $T^{-1}(X) \neq \emptyset$ ) and, obviously, reduces to a one-element set in case $T$ is an embedding. Moreover it is well known that the concrete category $\left(\mathrm{C}_{F}, T_{F, G}\right)$ (resp. $\left.(\mathrm{C}, T)\right)$ is fibre-complete (see e.g. the proof of [2, Proposition 21.11] for a description of the meets). Therefore $T_{F, G}^{-1}(X, \xi)$ (resp. $\left.T^{-1}(X)\right)$ is a (possibly large) complete lattice, i.e., every subclass thereof 
has a join and a meet. Let $(A, \alpha) \in T_{F, G}^{-1}(X, \xi)$. Then $T_{F, G}((A, \alpha))=(X, \xi)$, i.e., $(T(A), T(\alpha))=(X, \xi)$. Thus $A \in T^{-1}(X)$. A mapping $f:(A, \leq) \rightarrow(B, \leq)$ between posets is called an order embedding if $a \leq a^{\prime} \Leftrightarrow f(a) \leq f\left(a^{\prime}\right)$, for all $a, a^{\prime} \in A$, i.e. $f$ is both order-preserving and order-reflecting; see e.g. [13]. By extending this terminology to correspondences between preordered classes, one has:

Proposition 4.6. Under the assumptions of Theorem 4.1, the restriction-corestriction $\Phi_{(X, \xi)}: T_{F, G}^{-1}(X, \xi) \rightarrow T^{-1}(X)$ of the forgetful functor $U_{F}: C_{F} \rightarrow C$ is an injective order embedding which reflects (in)discrete objects.

Proof. Assume that $(A, \alpha) \leq(B, \beta)$ in $T_{F, G}^{-1}(X, \xi)$. Then there exists a morphism $\varphi:(A, \alpha) \rightarrow(B, \beta)$ such that $T_{F, G}(\varphi)=i d_{(X, \xi)}$. Therefore $T(A)=X=T(B)$ and $\varphi: A \rightarrow B$ is a morphism in $\mathrm{C}$ such that $T(\varphi)=i d_{X}$. Hence $A \leq B$ in $T^{-1}(X)$. Now assume that $(A, \alpha),(B, \beta) \in T_{F, G}^{-1}(X, \xi)$ are such that $\Phi_{(X, \xi)}(A, \alpha) \leq$ $\Phi_{(X, \xi)}(B, \beta)$ in $T^{-1}(X)$. Then there exists a unique morphism $\varphi: A \rightarrow B$ in $\mathrm{C}$ such that $T(\varphi)=i d_{X} \cdot \varphi$ is in fact a morphism in $\mathrm{C}_{F}$ from $(A, \alpha)$ to $(B, \beta)$. Indeed, one has

$$
\begin{aligned}
T(\beta \circ \varphi) & =T(\beta) \circ T(\varphi) \\
& =T(\beta) \circ i d_{X} \\
& =T(\beta)
\end{aligned}
$$

$$
\begin{aligned}
T(F(\varphi) \circ \alpha) & =T(F(\varphi)) \circ T(\alpha) \\
& =G(T(\varphi)) \circ T(\alpha) \\
& =i d_{G(X)} \circ T(\alpha) \\
& =T(\alpha) .
\end{aligned}
$$

Now $T(\beta)=\xi=T(\alpha)$ and $T$ is faithful. Thus $\beta \circ \varphi=F(\varphi) \circ \alpha$. Moreover $T_{F, G}(\varphi)=i d_{(X, \xi)}$. Hence $(A, \alpha) \leq(B, \beta)$. Although $U_{F}$ need not be injective on objects, $\Phi_{(X, \xi)}$ is injective. Indeed, let $(A, \alpha),(B, \beta) \in T_{F, G}^{-1}(X, \xi)$ with $\Phi_{(X, \xi)}(A, \alpha)=\Phi_{(X, \xi)}(B, \beta)$. Then $A=B$ and $T_{F, G}(A, \alpha)=T_{F, G}(B, \beta)$. Thus $A=B$ and $T(\alpha)=T(\beta)$ and since $T$ is faithful, it follows that $\alpha=\beta$ so that $(A, \alpha)=(B, \beta)$. The reflection of (in)discrete objects follows straightforwardly from Theorem 3.10 by observing that when $T_{F, G}^{-1}(X, \xi)$ and $T^{-1}(X)$ are viewed as full concrete subcategories of $C_{F}$ and $C$ over $\mathrm{X}_{G}$ and $\mathrm{X}$ with the inclusions $I: T_{F, G}^{-1}(X, \xi) \rightarrow C_{F}$ and $J: T^{-1}(X) \rightarrow C$, respectively, one has $J \circ \Phi_{(X, \xi)}=\Phi_{(X, \xi)} \circ I$.

4.2. M-topological categories of coalgebras. We find conditions under which the functor $T_{F, G}$ is Strong $\mathfrak{M}$ ono-topological.

Lemma 4.7. Assume that $X$ is an (Epi, $\mathfrak{S}$ trong $\mathfrak{M}$ ono)-category and $(C, T)$ is a concrete $\mathfrak{S}$ trong $\mathfrak{M}$ ono-topological category over $X, G: X \rightarrow X$ is a functor such that $G \circ T=T \circ F$ and $F$ preserves $T$-initial sources. Then every $T_{F, G}$-structured 
strong mono-source in $X_{G}$ carried by a strong mono-source in $X$ has a unique $T_{F, G^{-}}$ initial lift.

Proof. Let $\left(\varphi_{i}:(X, \xi) \rightarrow T_{F, G}\left(\left(A_{i}, \alpha_{i}\right)\right)\right)_{i \in I}$ be a strong mono-source in $\mathrm{X}_{G}$ such that $\left(\varphi_{i}: X \rightarrow T\left(A_{i}\right)\right)_{i \in I}$ is a strong mono-source in $\mathrm{X}$. By the Strong Monotopologicity of $T$, it has a unique $T$-initial lift $\left(\overline{\varphi_{i}}: C \rightarrow A_{i}\right)_{i \in I}$ in C. We have $T\left(\overline{\varphi_{i}}\right)=\varphi_{i}$ for each $i \in I$ and $\left(\alpha_{i} \circ \overline{\varphi_{i}}: C \rightarrow F\left(A_{i}\right)\right)_{i \in I}$ is a source in C. On the other hand, $F$ preserves $T$-initial sources. Thus, $\left(F\left(\overline{\varphi_{i}}\right): F(C) \rightarrow F\left(A_{i}\right)\right)_{i \in I}$ is a $T$-initial source in C. Moreover, since $\varphi_{i}:(X, \xi) \rightarrow\left(T\left(A_{i}\right), T\left(\alpha_{i}\right)\right)$ is a morphism in $\mathrm{X}_{G}$ for each $i \in I$, it follows that $\xi: X \rightarrow G(X)$ and $T\left(\alpha_{i}\right) \circ \varphi_{i}=G\left(\varphi_{i}\right) \circ \xi$, i.e., $\xi: T(C) \rightarrow T(F(C))$ and $T\left(\alpha_{i}\right) \circ T\left(\overline{\varphi_{i}}\right)=G\left(T\left(\overline{\varphi_{i}}\right)\right) \circ \xi$, for each $i \in I$. Thus the $T$-initiality of the source $\left(F\left(\overline{\varphi_{i}}\right): F(C) \rightarrow F\left(A_{i}\right)\right)_{i \in I}$ in $C$ yields a unique morphism $\bar{\xi}: C \rightarrow F(C)$ such that $T(\bar{\xi})=\xi$ and $\alpha_{i} \circ \overline{\varphi_{i}}=F\left(\overline{\varphi_{i}}\right) \circ \bar{\xi}$, for each $i \in I$. The rest of the proof is as in that of Theorem 4.1 where the faithfulness of $T$ given by its topologicity via Remark 3.2 is guaranteed by the fact that $(\mathrm{C}, T)$ is concrete over $\mathrm{X}$.

Theorem 4.8. Assume that in addition to the assumptions in Lemma 4.7 the functor $G$ preserves strong mono-sources. Then the functor $T_{F, G}$ is $\mathfrak{S}$ trong $\mathfrak{M}$ onotopological.

Proof. By Corollary 2.5 the category $\mathrm{X}_{G}$ is an ( $\mathcal{E}$ pi,Strong $\mathfrak{M}$ ono)-category. Moreover by Lemma 2.4 strong mono-sources in $\mathrm{X}_{G}$ are precisely sources that are carried by strong mono-sources in X. Thus by Lemma 4.7, every $T_{F, G}$-structured Strong $\mathfrak{M}$ ono-source has a unique $T_{F, G}$-initial lift.

Corollary 4.9. Under the assumptions of Theorem 4.8, $\left(C_{F}, T_{F, G}\right)$ is an epireflective concrete subcategory of some topological category over $X_{G}$.

Proof. This follows from Theorem 4.8 and [2, Theorem 21.40].

Acknowledgement. The author would like to thank the anonymous referee who provided him with very valuable comments and suggestions.

\section{References}

[1] J. Adámek, Introduction to coalgebra, Theory Appl. Categ., 14(8) (2005), 157199.

[2] J. Adámek, H. Herrlich and G. E. Strecker, Abstract and Concrete Categories, Pure and Applied Mathematics (New York), A Wiley-Interscience Publication, John Wiley \& Sons, Inc., New York, 1990. 
[3] J. Adámek and H.-E. Porst, On varieties and covarieties in a category, Coalgebraic methods in computer science (Genova, 2001), Math. Structures Comput. Sci., 13(2) (2003), 201-232.

[4] P. Antoine, Étude élémentaire des catégories d'ensembles structurés, Bull. Soc. Math. Belg., 18 (1966), 142-164.

[5] G. C. L. Brümmer, Topological categories, Topology Appl., 18(1) (1984), 27-41.

[6] R. Garner, Topological functors as total categories, Theory Appl. Categ., 29(15) (2014), 406-422.

[7] H. Herrlich, Topological functors, General Topology and Appl., 4 (1974), 125142.

[8] H. Herrlich, Initial completions, Math. Z., 150(2) (1976), 101-110.

[9] H. Herrlich and G. E. Strecker, Semi-universal maps and universal initial completions, Pacific J. Math., 82(2) (1979), 407-428.

[10] Y. H. Hong, Studies on Categories of Universal Topological Algebras, Ph.D. Thesis, McMaster Univ., Canada, 1974.

[11] Y. H. Hong, On initially structured functors, J. Korean Math. Soc., 14(2) (1977/78), 159-165.

[12] P. Johnstone, J. Power, T. Tsujishita, H. Watanabe and J. Worrell, On the structure of categories of coalgebras, Coalgebraic methods in computer science (Lisbon, 1998), Theoret. Comput. Sci., 260(1-2) (2001), 87-117.

[13] V. Laan and S. Nasir, On mono- and epimorphisms in varieties of ordered algebras, Comm. Algebra, 43(7) (2015), 2802-2819.

[14] P. Lundström, Weak topological functors, J. Gen. Lie Theory Appl., 2(3) (2008), 211-215.

[15] L. D. Nel, Initially structured categories and Cartesian closedness, Canadian J. Math., 27(6) (1975), 1361-1377.

[16] G. Preuss, Point separation axioms, monotopological categories and MacNeille completions, Category Theory at Work (Bremen, 1990), H. Herrlich, H.-E. Porst (eds.), Res. Exp. Math., Heldermann, Berlin, 18 (1991), 47-55.

[17] F. Schwarz, Funktionenräume und Exponentiale Objekte in Punktetrennen Initialen Kategorien, Ph.D. Thesis, Univ. Bremen, 1983.

[18] D. Zangurashvili, Effective codescent morphisms, amalgamations and factorization systems, J. Pure Appl. Algebra, 209(1) (2007), 255-267. 


\section{Maurice Kianpi}

Laboratory of Algebra, Geometry and Applications

Department of Mathematics

Faculty of Science

University of Yaounde 1

P.O. Box 812, Yaounde, Cameroon

e-mail: mkianpi@uy1.uninet.cm; mkianpi@yahoo.com 